Original Article

\title{
Antioxidant status and their enhancements strategies for water stress tolerance in chickpea
}

\author{
Status antioxidante e suas estratégias de aprimoramento para tolerância ao estresse \\ hídrico no grão-de-bico
}

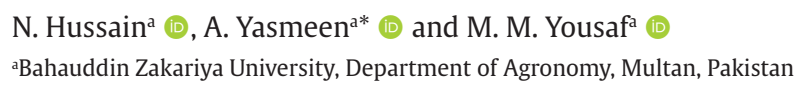

\begin{abstract}
Water stress executes severe influences on the plant growth and development through modifying physio-chemical properties. Therefore, a field experiment was designed to evaluate the antioxidant status and their enhancements strategies for water stress tolerance in chickpea on loam and clay loam soils under agro-ecological conditions of Arid Zone Research Institute, Bahawalpur $\left(29.3871^{\circ} \mathrm{N}, 71.653^{\circ} \mathrm{E}\right)$ and Cholistan farm near Derawer $\left(28.19^{\circ} \mathrm{N}, 71.80^{\circ} \mathrm{E}\right)$ of Southern Punjab, Pakistan during winter 2014-15. Experimental treatments comprised of two chickpea cultivars i.e. Bhakhar 2011 (drought tolerant) and DUSHT (drought sensitive), two water stress levels i.e. water stress at flowering stage and water stress at flowering + pod formation + grain filling stage including well watered (control) and two exogenous application of osmoprotectants i.e. glycine betaine (GB) $20 \mathrm{ppm}$ and proline $10 \mathrm{uM}$ including distilled water (control). Results indicated that water stress at various growth stages adversely affects the growth, yield and quality attributes of both chickpea cultivars. Exogenous application of GB and proline improved the growth, yield and quality parameters of both chickpea cultivars even under water stress conditions. However, superior results were obtained with exogenously applied GB on Bhakhar 2011 under well-watered conditions. Similarly, foliar spray of GB on chickpea cultivar Bhakhar 2011 under stress at flowering + pod formation + grain filling stage produced maximum superoxide dismutase, peroxidase and catalase contents. These results suggested that application of GB mitigates the adverse effects of water stress and enhanced tolerance in chickpea mainly due to higher antioxidant enzymes activity, demonstrating the protective measures of plant cells in stress condition. Hence, antioxidants status might be a suitable method for illustrating water stress tolerance in chickpea.
\end{abstract}

Keywords: superoxide dismutase, peroxidase, catalase, glycine betaine, proline.

\section{Resumo}

O estresse hídrico exerce fortes influências no crescimento e no desenvolvimento das plantas, modificando as propriedades físico-químicas. Portanto, a presente atividade de pesquisa foi projetada para avaliar o status antioxidante e suas estratégias de aprimoramento para tolerância ao estresse hídrico no grão-de-bico em condições agroecológicas, no Instituto de Pesquisa da Zona Árida, Bahawalpur $\left(29.3871^{\circ} \mathrm{N}, 71.653^{\circ} \mathrm{E}\right)$ e fazenda do Cholistan, perto de Derawer $\left(28.19^{\circ} \mathrm{N}, 71,80^{\circ} \mathrm{E}\right)$, no sul de Punjab, Paquistão, durante Rabi 2014-15. Tratamentos experimentais compostos de dois genótipos de grão-de-bico, como Bhakhar 2011 (tolerante à seca) e DUSHT (sensível à seca), dois níveis de estresse hídrico, ou seja, estresse hídrico no estágio de floração, estresse hídrico na fase de floração e estresse hídrico na fase de floração + formação de vagem + estágio de enchimento de grãos, incluindo água bem controlada (controle) e duas aplicações exógenas de osmoprotetores, isto é, glicina betaína 20 ppm e prolina 10 uM, incluindo água destilada (controle). Os resultados indicaram que o estresse hídrico em vários estágios de crescimento afeta negativamente os atributos de crescimento, rendimento e qualidade de ambas as cultivares de grão-de-bico. A aplicação exógena de glicina betaína e prolina melhorou os parâmetros de crescimento, rendimento e qualidade de ambos os genótipos de grão- de-bico, mesmo sob condições de estresse hídrico. No entanto, resultados superiores foram obtidos com glicina betaína aplicada exogenamente em Bhakhar 2011, em condições bem regadas. Além disso, o spray foliar de glicina betaína na cultivar de grão-de-bico Bhakhar 2011, sob estresse na fase de floração + formação de vagem + enchimento de grãos, produziu o máximo de superóxido dismutase, peroxidase e catalase. Esses resultados sugeriram que a aplicação de glicina betaína atenua os efeitos adversos do estresse hídrico e aumenta a tolerância no grão-de- bico, principalmente pela maior atividade de enzimas antioxidantes, demonstrando medidas protetoras das células vegetais em condições de estresse. Portanto, o status de antioxidantes pode ser um método adequado para ilustrar a tolerância ao estresse hídrico no grão-de-bico.

Palavras-chave: superóxido dismutase, peroxidase, catalase, glicina betaína, prolina.

*e-mail: azra.yasmeen@bzu.edu.pk

Received: May 9, 2020 - Accepted: October 9, 2020

This is an Open Access article distributed under the terms of the Creative Commons Attribution License, which permits unrestricted use, distribution, and reproduction in any medium, provided the original work is properly cited. 


\section{Introduction}

Chickpea (Cicer arietinum L.) belongs to leguminosae family, plays an important role in human nutrition for large population sectors in the developing world and is considered as a healthy food in many developed countries (Merga and Haji, 2019). In addition to its high protein content (20-28\%) and carbohydrates (60-65\%), chickpea is a good source of essential amino acids and minerals (Merga and Haji, 2019). Moreover, chickpea is a crucial source of fodder and also fixes atmospheric nitrogen through symbiotic nitrogen fixation, hence plays a key role in enhancing and sustaining the overall productivity of the cropping systems (Khaitov et al., 2016).

Chickpea is traditionally cultivated in arid and semiarid tropical regions under rain-fed condition on residual moisture preserved after monsoon rains (during July and August). It is susceptible to terminal water stress due to depletion of stored soil moisture towards maturation, and experiences up to 50\% yield loss (Sinha et al., 2019). Plants experience water stress either when the water supply to roots is interrupted or when transpiration rate becomes very high. When plants are subjected to water stress conditions, show lot of morpho-physiological and biochemical changes (Reddy and Raghavendra, 2006). Under such conditions, some reactive oxygen species (ROS) are produced (MunneBosch and Penuelas, 2003). While the amount of ROS production beats the antioxidant defense competency of the cell, ensuing in cellular damages (Almeselmani et al., 2006). Imbalance between the production of ROS and the antioxidant defense inhibits the growth and photosynthetic abilities of plants and injure the cellular components of proteins, membrane lipids and nucleic acids under water stress conditions (Peifang et al., 2015).

Plants adapted to water stress accumulate particular molecules, which have a cryo-protective role (Farooq et al., 2009). Glycine betaine (GB) is one such cryo-protective solute that stabilizes membranes and photosynthetic apparatus, detoxification of ROS, cellular osmotic adjustment and protects the activities of proteins and enzymes involved in the antioxidant defense system under water stress conditions (Chen and Murata, 2011). GB is an organic compound present in plants, that is anamphoteric quaternary amine, plays an imperative part as a compatible solute in plants under water stress conditions (Ashraf and Foolad, 2007). It accumulates in response to water stress in the leaves of some plants that may confer tolerance to the osmotic stress (Farooq et al., 2008). It is primarily abundant in chloroplast where it plays a dynamic role in adjustment and protection of thylakoid lamella, thereby improving the photosynthetic efficiency under water stress conditions (Wang et al., 2010).

In addition to $\mathrm{GB}$, exogenous application of proline is attaining important consideration to cope with water stress (Sadak and Ahmed, 2016). Proline is a proteinogenic amino acid, extremely important for numerous vital metabolic processes within the plant tissues. At optimum level, it has various roles such as osmoprotectant, balance of redox status, storage of carbon and nitrogen, turgor generation, maintenance the structure of proteins and cytosolic $\mathrm{pH}$, acting as a part of stress signal, an inhibitor of lipid membrane peroxidation and antioxidant as effective quencher of ROS formed under stress conditions in plants (Hayat et al., 2012). Proline can act as a signaling molecule to influence cell proliferation, modify mitochondrial functions and trigger specific gene expression, which can be vital for plant recovery from water stress conditions (Hayat et al., 2012). Limited number of studies was conducted on the influence of GB and proline on the chickpea productivity under stress conditions. It is hypothesized that exogenous application of GB and proline may positively affect the physiological attributes of chickpea and improved the growth and productivity under water stress conditions. Keeping these facts in view, the present investigation was therefore undertaken to study the role of osmoprotectants to mitigate the adverse effect of water stress on chickpea genotypes through the modulation of antioxidant enzymes status.

\section{Materials and Methods}

\subsection{Experimental detail}

The present research activity was designed to evaluate the antioxidant status and their enhancements strategies for water stress tolerance in chickpea under agro-ecological conditions of Arid Zone Research Institute, Bahawalpur $\left(29.3871^{\circ} \mathrm{N}, 71.653^{\circ} \mathrm{E}\right)$ and Cholistan farm near Derawer $\left(28.19^{\circ} \mathrm{N}, 71.80^{\circ} \mathrm{E}\right)$ of Southern Punjab, Pakistan during winter 2014-15. Experimental treatments comprised of two chickpea genotypes i.e. Bhakhar 2011 (drought tolerant) and DUSHT (drought sensitive), two water stress levels i.e. Water stress at flowering stage and water stress at flowering + pod formation + grain filling stage including well watered (control) and two exogenous application of osmoprotectants i.e. glycine betaine 20 ppm (Bardhan et al., 2007) and proline 10 uM (Kaur et al., 2011) including distilled water (control). Foliar spray was applied twice at $50 \%$ flowering and at $50 \%$ pod formation stage. Field experiment was arranged in a Randomized Complete Block Design with factorial experiment having three repeats.

Prior to sowing, composite soil samples were collected from experimental areas at the depth of $0-30 \mathrm{~cm}$ and examined for physico-chemical features. The soil texture of experimental areas were loam and clay loam, having $\mathrm{pH} 8.2$ and 7.9, organic matter 0.77 and $0.93 \%$, EC 2.5 and $2.1 \mathrm{dS} \mathrm{m}^{-1}$, total nitrogen 0.034 and $0.049 \%$, available phosphorus 6.3 and 4.5 ppm and exchangeable potassium 192 and 94 ppm in Bahawalpur and Cholistan, respectively.

\subsection{Crop husbandry}

Pre-soaking irrigation was applied before seedbed preparation. When the soil reached to a workable moisture level, seedbed was prepared by cultivating the field twice with tractor-mounted cultivator each followed by planking during winter 2014-15. Seeds of both chickpea cultivars was sown by hand drill using $60 \mathrm{~kg} \mathrm{ha}^{-1}$ seed rate by keeping row to row distance of $25 \mathrm{~cm}$ and plant to plant distance $10 \mathrm{~cm}$ during last week of October 2014. Seeds were placed at 3 to $5 \mathrm{~cm}$ depth in each row. The seedlings were thinned out by maintaining one plant per 
hill at 15 days after sowing. Recommended dose of Urea @ $20 \mathrm{Kg} \mathrm{N}^{-1}$ and Di-ammonium phosphate (DAP) @ $22 \mathrm{Kg}$ $\mathrm{P} \mathrm{ha}^{-1}$ was applied at the time of seedbed preparation. Plant protection measures and agronomic practices were kept uniform and normal for all the experimental units throughout the growing season.

\subsection{Data collection}

Thirty days after sowing, ten randomly selected plants were tagged to record the secondary branches per plant, number of pods per plant and 100 grain weight.

Growth parameters were examined three times during growing seasons at both locations. Data regarding CGR was recorded by adopting the following Formula 1 of Watson (1952).

$$
\operatorname{CGR}\left(\mathrm{g} \mathrm{m}^{-2} \text { day }^{-1}\right)=\left(\mathrm{W}_{2}-\mathrm{W}_{1}\right) /\left(\mathrm{T}_{2}-\mathrm{T}_{1}\right)
$$

whereas, $W_{1}$ and $W_{2}$ is the oven-dried weight at first and second sampling, $\mathrm{T}_{1}$ and $\mathrm{T}_{2}$ is the time of first and second sampling.

Relative growth rate (RGR) was calculated by using Equation 2 proposed by Radford (1967).

$$
\operatorname{RGR}\left(\mathrm{g} \mathrm{g}^{-1} \text { day }^{-1}\right)=\left(\operatorname{LnW}_{2}-\mathrm{LnW} 1\right) /\left(\mathrm{T}_{2}-\mathrm{T}_{1}\right)
$$

whereas, $L n=$ Natural Log, $W_{1}$ and $W_{2}$ is the oven-dried weight at first and second sampling, $\mathrm{T}_{1}$ and $\mathrm{T}_{2}$ is the time of first and second sampling.

Total soluble protein $\left(\mathrm{mg} \mathrm{g}^{-1}\right)$ was determined according to procedure followed by Bradford (1976). The $\mathrm{K}^{+}$content in leaves were determined by flame photometer (Richards, 1954). Superoxide dismutase (IU min ${ }^{-1} \mathrm{mg}^{-1}$ protein) was calculated by adopting the methodology followed by (Giannopolitis and Ries, 1977). Moreover, peroxidase (IU min ${ }^{-1} \mathrm{mg}^{-1}$ protein) and catalase (IU min ${ }^{-1} \mathrm{mg}^{-1}$ protein) was calculated by adopting the procedure followed by Chance and Maehly (1955). $1 \mathrm{~m}^{2}$ from each experimental unit was harvested and threshed manually to record the grain yield per unit area on $11^{\text {th }}$ April 2015.

\subsection{Statistical analysis}

All the collected data was analyzed by using the MSTAT software. The Duncan's Multiple Range test (DMRt) was used to compare the differences among treatment means at $5 \%$ probability level (Steel et al., 1997).

\section{Results}

\subsection{Crop growth rate (CGR) $\left(\mathrm{g} \mathrm{m}^{-2}\right.$ day $\left.^{-1}\right)$}

CGR is the rate of dry matter accumulation per unit ground area. Exogenous application of osmoprotectants on chickpea genotypes under water stress conditions significantly affected the CGR recorded at different growth stages at both locations (Figure 1a and 1b). The crop growth rate (CGR) followed an increasing trend in the initial stages of crop growth and decreased thereafter. Exogenous application of glycine betaine on chickpea cultivar Bhakhar 2011 cultivated under well-watered condition produced significantly higher CGR at all growth stages. Chickpea genotype DUSHT cultivated under water stress at flowering + pod formation + grain filling stage produced minimum CGR throughout the growing season.

\subsection{Relative Growth Rate (RGR) $\left(\mathrm{g} \mathrm{g}^{-1}\right.$ day $\left.^{-1}\right)$}

RGR is the increase in dry mass per unit of plant mass over a specified period of time. Exogenous application of osmoprotectants on chickpea genotypes under water stress conditions significantly affected the RGR recorded at different growth stages at both locations (Figure 2a and 2b). Exogenous application of glycine betaine on chickpea cultivar Bhakhar 2011 cultivated under well-watered condition produced significantly higher RGR at all growth stages. Chickpea cultivar DUSHT cultivated under water stress at flowering + pod formation + grain filling stage produced minimum RGR throughout the growing season at both locations.

\subsection{Number of secondary branches per plant}

Secondary branches develop at buds located on the primary branches and determines the total number of leaves, and hence the total photosynthetic area. Comparison of the treatment means showed that exogenous application of osmoprotectants on chickpea genotypes under water stress at different growth stages significantly affected the number of secondary branches per plant at both locations (Table 1). Exogenous application of glycine betaine on Bhakhar 2011 cultivated under well watered conditions produced maximum number of secondary branches per plant against the minimum was observed from DUSHT cultivated under water stress condition at flowering + pod formation + grain filling stage.

\subsection{Number of pods per plant}

Number of pods per plant as the major yield parameters reflects the plant performance during previous growth stages, which depend mainly on the vigorous of vegetative growth and flowering status. Comparison of the treatment means showed that exogenous application of osmoprotectants on chickpea genotypes under water stress at different growth stages significantly affected the number of pods per plant at both locations (Table 1). Exogenous application of glycine betaine on Bhakhar 2011 cultivated under well watered conditions produced maximum number of pods per plant against the minimum was observed from DUSHT cultivated under water stress condition at flowering + pod formation + grain filling stage.

\subsection{0 grains weight ( $g$ )}

Grains weight is crucial yield boosting attribute and has been given due consideration during varietal assessing process. Comparison of the treatment means showed that exogenous application of osmoprotectants on chickpea genotypes under water stress at different growth stages significantly affected the 100 grains weight at both locations (Table 1). Exogenous application of glycine betaine on Bhakhar 2011 cultivated under well watered 

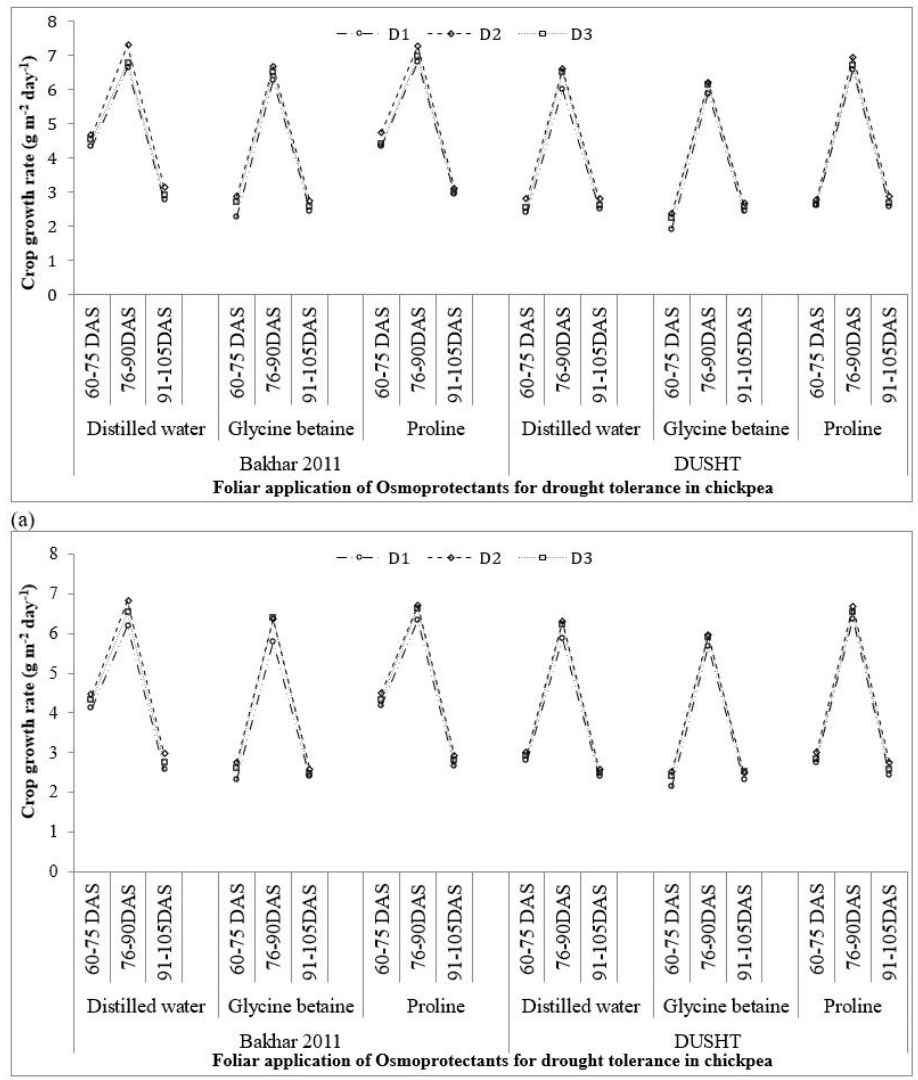

(b)

Figure 1. (a) Influence of exogenous application of osmoprotectants on crop growth rate $\left(\mathrm{g} \mathrm{m}^{-2} \mathrm{day}^{-1}\right)$ of chickpea genotypes in Bahawalpur; (b) Influence of exogenous application of osmoprotectants on crop growth rate $\left(\mathrm{g} \mathrm{m}^{-2} \mathrm{day}^{-1}\right)$ of chickpea genotypes in Cholistan. Whereas D1 = well watered; D2= Drought at flowering+ pod formation + grain filling stage; D3= Drought at flowering stage; DAS, Days after sowing.

conditions produced maximum 100 grains weight against the minimum was observed from DUSHT cultivated under water stress condition at flowering + pod formation + grain filling stage.

\subsection{Grain yield $\left(\mathrm{kg} \mathrm{ha}^{-1}\right)$}

Comparison of the treatment means showed that exogenous application of osmoprotectants on chickpea genotypes under water stress at different growth stages significantly affected the final grain yield at both locations (Table 2). Exogenous application of glycine betaine on Bhakhar 2011 cultivated under well watered conditions produced maximum grain yield against the minimum was observed from DUSHT cultivated under water stress at flowering + pod formation + grain filling stage.

\subsection{Leaf total soluble protein $\left(\mathrm{mg}^{-1}\right)$}

Comparison of the treatment means showed that exogenous application of osmoprotectants on chickpea genotypes under water stress at different growth stages significantly affected the leaf total soluble protein at both locations (Table 2). Exogenous application of glycine betaine on Bhakhar 2011 cultivated under well watered conditions produced maximum leaf total soluble protein. While minimum leaf total soluble protein was observed from DUSHT cultivated under water stress condition at flowering + pod formation + grain filling stage.

\subsection{Leaf $K^{+}$contents}

Comparison of the treatment means showed that exogenous application of osmoprotectants on chickpea genotypes under water stress at different growth stages significantly affected the leaf $\mathrm{K}^{+}$contents at both locations (Table 2). Exogenous application of glycine betaine on Bhakhar 2011 cultivated under water stress at grain filling stage produced maximum leaf $\mathrm{K}^{+}$contents. While minimum leaf $\mathrm{K}^{+}$contents was observed from DUSHT cultivated under water stress condition at flowering + pod formation + grain filling stage.

\subsection{Superoxide dismutase (SOD) (IU $\mathrm{min}^{-1} \mathrm{mg}^{-1}$ protein)}

Superoxide dismutase (SOD) establishes the first line of protection through detoxification of superoxide radicals, hence sustain membranes of plant tissue (Sairam and Saxena, 2000). Comparison of the treatment means showed that exogenous application of osmoprotectants on chickpea genotypes under water stress at different growth stages significantly affected the SOD at both locations (Table 3 ). Exogenous application of glycine betaine on Bhakhar 2011 cultivated under water stress at flowering + pod 


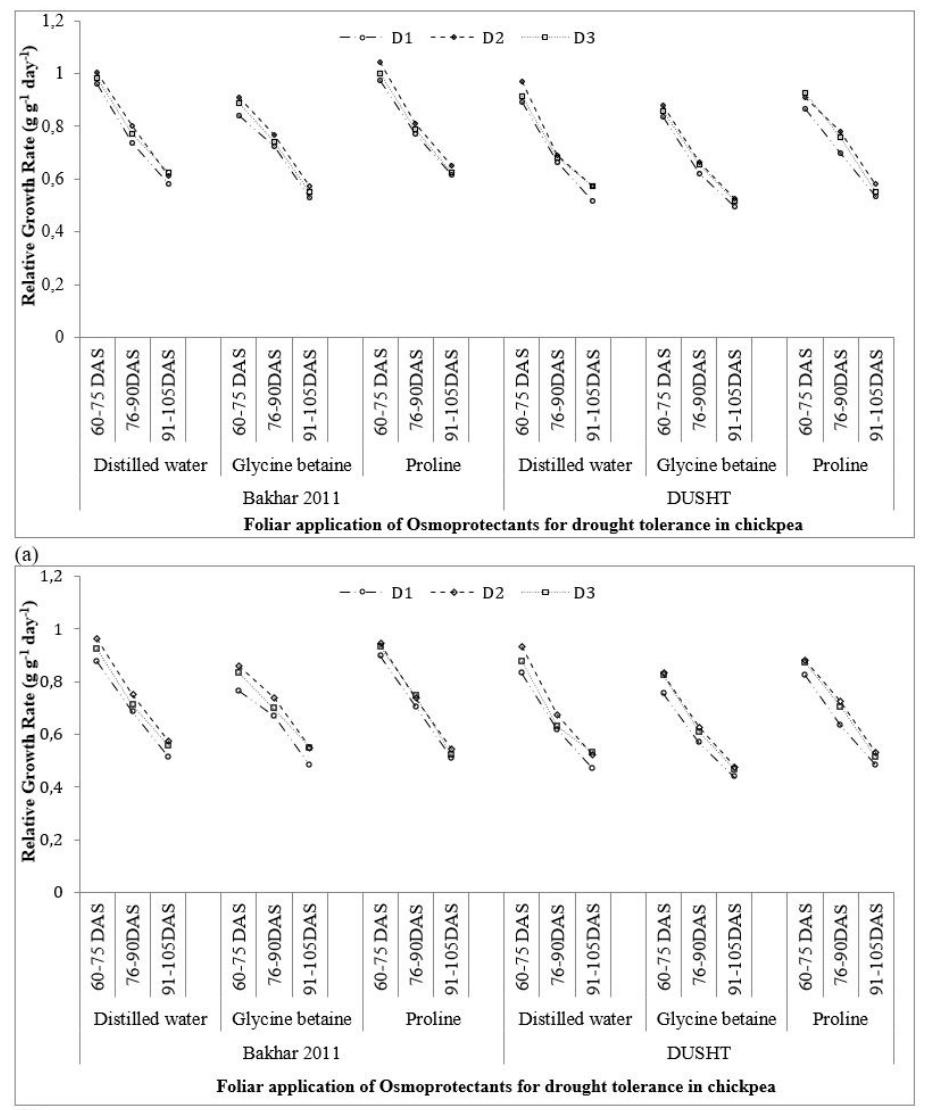

(b)

Figure 2. (a) Influence of exogenous application of osmoprotectants on relative growth rate $\left(\mathrm{g} \mathrm{g}^{-1}\right.$ day $\left.^{-1}\right)$ of chickpea genotypes in Bahawalpur; (b) Influence of exogenous application of osmoprotectants on relative growth rate $\left(\mathrm{g} \mathrm{g}^{-1}\right.$ day $\left.^{-1}\right)$ of chickpea genotypes in Cholistan. Whereas D1 = well watered; D2= Drought at flowering+ pod formation + grain filling stage; D3= Drought at flowering stage; DAS, Days after sowing.

formation + grain filling stage produced maximum SOD. While minimum SOD was observed from DUSHT cultivated under well-watered condition.

\subsection{Peroxidase (POD) (IU min-1 $\mathrm{mg}^{-1}$ protein)}

Comparison of the treatment means showed that exogenous application of osmoprotectants on chickpea genotypes under water stress at different growth stages significantly affected the POD at both locations (Table 3). Exogenous application of glycine betaine on Bhakhar 2011 cultivated under water stress at flowering + pod formation + grain filling stage produced maximum POD. While minimum POD was observed from DUSHT cultivated under well-watered condition.

\subsection{Catalase (CAT) (IU min ${ }^{-1} \mathrm{mg}^{-1}$ protein)}

Catalase (CAT) eradicates $\mathrm{H}_{2} \mathrm{O}_{2}$ by breaking it down directly to water and oxygen. CAT and POD are the essential enzymes involved in regulation of intracellular level of $\mathrm{H}_{2} \mathrm{O}_{2}$. Comparison of the treatment means showed that exogenous application of osmoprotectants on chickpea genotypes under water stress at different growth stages significantly affected the CAT at both locations (Table 3 ).
Exogenous application of glycine betaine on Bhakhar 2011 cultivated under water stress at flowering + pod formation + grain filling stage produced maximum CAT. While minimum CAT was observed from DUSHT cultivated under well-watered condition.

\section{Discussion}

Water stress inhibited the growth and development chickpea of plants and showed several morphological and physiological modifications (Raheleh et al., 2012). In present study, water stress at flowering + pod formation + grain filling stage significantly reduced the crop and relative growth rate of chickpea cultivars at both locations. Crop growth is accomplished through cell division, cell enlargement and cell differentiation and involves genetic, physiological, ecological and morphological events and their complex interactions. Under water stress conditions, cell elongation of plants can be inhibited by interruption of water flow from the xylem to the surrounding elongating cells (Awari et al., 2017). However, application of osmoprotectants improves the crop growth both under stressed and well-watered 
Table 1. Influence of exogenous application of osmoprotectants on secondary branches, number of pods and 100-grain weight (g) of chickpea.

\begin{tabular}{|c|c|c|c|c|c|c|c|c|}
\hline \multirow{2}{*}{$\begin{array}{c}\text { Chickpea } \\
\text { genotypes }\end{array}$} & \multirow{2}{*}{$\begin{array}{c}\text { Water } \\
\text { stress } \\
\text { level }\end{array}$} & \multirow{2}{*}{$\begin{array}{c}\text { Foliar } \\
\text { spray }\end{array}$} & \multicolumn{2}{|c|}{$\begin{array}{l}\text { Secondary branches per } \\
\text { plant }\end{array}$} & \multicolumn{2}{|c|}{$\begin{array}{c}\text { Number of pods per } \\
\text { plant }\end{array}$} & \multicolumn{2}{|c|}{ 100-grain weight (g) } \\
\hline & & & Bahawalpur & Cholistan & Bahawalpur & Cholistan & Bahawalpur & Cholistan \\
\hline \multirow[t]{9}{*}{$\begin{array}{c}\text { Bhakhar } \\
2011\end{array}$} & \multirow[t]{3}{*}{$\begin{array}{c}\text { Well } \\
\text { watered }\end{array}$} & $\begin{array}{l}\text { Distilled } \\
\text { water }\end{array}$ & $9.50 \mathrm{ac}$ & $9.76 \mathrm{ac}$ & $26.27 \mathrm{bd}$ & $28.46 \mathrm{ad}$ & $202.60 \mathrm{ac}$ & $204.94 \mathrm{ab}$ \\
\hline & & $\begin{array}{l}\text { Glycine } \\
\text { betaine }\end{array}$ & $9.85 \mathrm{a}$ & $10.16 \mathrm{a}$ & $31.16 \mathrm{a}$ & $31.52 \mathrm{a}$ & $208.19 a$ & $209.86 a$ \\
\hline & & Proline & $9.27 \mathrm{ad}$ & $9.95 \mathrm{ab}$ & $29.10 \mathrm{ac}$ & $30.44 \mathrm{ab}$ & $206.33 \mathrm{ab}$ & $205.67 \mathrm{ab}$ \\
\hline & \multirow{3}{*}{$\begin{array}{l}\text { Water } \\
\text { stress at } \\
\text { flowering } \\
\text { + pod } \\
\text { formation } \\
\text { + grain } \\
\text { filling } \\
\text { stage }\end{array}$} & $\begin{array}{l}\text { Distilled } \\
\text { water }\end{array}$ & $8.18 \mathrm{df}$ & $8.51 \mathrm{eh}$ & $21.68 \mathrm{eg}$ & $24.40 \mathrm{cf}$ & $186.19 \mathrm{~cd}$ & $189.86 \mathrm{~cd}$ \\
\hline & & $\begin{array}{l}\text { Glycine } \\
\text { betaine }\end{array}$ & $8.93 \mathrm{ad}$ & $8.96 \mathrm{df}$ & $27.61 \mathrm{ad}$ & $28.27 \mathrm{ad}$ & $201.12 \mathrm{ac}$ & $202.70 \mathrm{ab}$ \\
\hline & & Proline & 8.31 ce & $8.71 \mathrm{dg}$ & $26.37 \mathrm{bd}$ & 26.72 ae & $197.37 \mathrm{ad}$ & $194.03 \mathrm{ad}$ \\
\hline & \multirow{3}{*}{$\begin{array}{c}\text { Water } \\
\text { stress at } \\
\text { flowering } \\
\text { stage }\end{array}$} & $\begin{array}{l}\text { Distilled } \\
\text { water }\end{array}$ & 8.61 be & $8.84 \mathrm{df}$ & $26.31 \mathrm{bd}$ & 26.69 ae & $198.63 \mathrm{ac}$ & $197.30 \mathrm{ac}$ \\
\hline & & $\begin{array}{l}\text { Glycine } \\
\text { betaine }\end{array}$ & $9.60 \mathrm{ab}$ & $9.36 \mathrm{bd}$ & $29.45 a b$ & $29.88 \mathrm{ac}$ & $206.90 \mathrm{ab}$ & $205.23 \mathrm{ab}$ \\
\hline & & Proline & $8.97 \mathrm{ad}$ & $9.04 \mathrm{df}$ & $28.09 \mathrm{ad}$ & $28.76 \mathrm{ad}$ & $201.35 \mathrm{ac}$ & $202.01 \mathrm{ab}$ \\
\hline \multirow[t]{10}{*}{ DUSHT } & \multirow[t]{3}{*}{$\begin{array}{c}\text { Well } \\
\text { watered }\end{array}$} & $\begin{array}{l}\text { Distilled } \\
\text { water }\end{array}$ & 8.45 be & $9.02 \mathrm{de}$ & 25.71 be & 26.37 ae & 194.18 ad & $196.51 \mathrm{ac}$ \\
\hline & & $\begin{array}{l}\text { Glycine } \\
\text { betaine }\end{array}$ & $8.90 \mathrm{ad}$ & $9.23 \mathrm{~cd}$ & 27.12 ad & $28.22 \mathrm{ad}$ & $199.73 \mathrm{ac}$ & $203.07 \mathrm{ab}$ \\
\hline & & Proline & 8.55 be & 9.13 ce & $25.50 \mathrm{bf}$ & 27.13 ae & $195.52 \mathrm{ad}$ & $198.76 \mathrm{ac}$ \\
\hline & \multirow{3}{*}{$\begin{array}{l}\text { Water } \\
\text { stress at } \\
\text { flowering } \\
\text { + pod } \\
\text { formation } \\
\text { + grain } \\
\text { filling } \\
\text { stage }\end{array}$} & $\begin{array}{l}\text { Distilled } \\
\text { water }\end{array}$ & $7.01 \mathrm{f}$ & $6.94 \mathrm{j}$ & $19.29 \mathrm{~g}$ & $20.12 \mathrm{f}$ & $163.32 \mathrm{e}$ & $169.98 \mathrm{e}$ \\
\hline & & $\begin{array}{l}\text { Glycine } \\
\text { betaine }\end{array}$ & $8.27 \mathrm{de}$ & $7.99 \mathrm{hi}$ & $24.28 \mathrm{df}$ & $23.62 \mathrm{df}$ & $186.17 \mathrm{~cd}$ & $182.84 \mathrm{ce}$ \\
\hline & & Proline & $8.19 \mathrm{df}$ & $8.05 \mathrm{gi}$ & $21.14 \mathrm{fg}$ & $21.81 \mathrm{ef}$ & $179.85 \mathrm{de}$ & $178.85 \mathrm{de}$ \\
\hline & \multirow{3}{*}{$\begin{array}{l}\text { Water } \\
\text { stress at } \\
\text { flowering } \\
\text { stage }\end{array}$} & $\begin{array}{l}\text { Distilled } \\
\text { water }\end{array}$ & $7.67 \mathrm{ef}$ & $7.83 \mathrm{i}$ & $24.83 \mathrm{cf}$ & $25.49 \mathrm{bf}$ & $185.16 \mathrm{~cd}$ & $178.56 \mathrm{de}$ \\
\hline & & $\begin{array}{l}\text { Glycine } \\
\text { betaine }\end{array}$ & $9.13 \mathrm{ad}$ & $8.96 \mathrm{df}$ & 26.45 bd & 27.14 ae & $195.71 \mathrm{ad}$ & $193.18 \mathrm{ad}$ \\
\hline & & Proline & 8.28 ce & $8.45 \mathrm{fi}$ & 25.89 be & 26.96 ae & 188.51 bd & $190.51 \mathrm{bd}$ \\
\hline & LSD $0.05 p=$ & & 1.2204 & 0.6690 & 4.3911 & 5.5971 & 18.481 & 16.937 \\
\hline
\end{tabular}

LSD= Least significant difference.

conditions. Exogenous application of GB on chickpea cultivar Bhakhar 2011 cultivated under well-watered conditions produced significantly higher crop and relative growth rate at all growth stages. Earlier studies showed the positive influence of GB on plant growth (Ashraf and Foolad, 2007). Foliar spray of GB can promptly penetrate through leaf surface and be easily transferred to other parts, where it would contribute to enhancement in water stress tolerance (Ashraf and Foolad, 2007). Foliarly applied GB induced tolerance might be credited to reducing of the osmotic potential due to net solute accumulation in response to water stress, which could assist to preserve the metabolic processes, contribute to growth of plants through maintaining turgor in cells and ultimately increase drought tolerance, thus maintaining higher photosynthetic activities (Hussain et al., 2009).

The results of present investigation revealed that the number of branches per plant was reduced significantly due to the water stress as compared to the well-watered condition. However, exogenous application of GB produced significantly higher number of branches plant even under water stress conditions. As it was earlier literature showed that GB application at early stage of crop was found to be more effective in ameliorating the negative impact of water 
Table 2. Influence of exogenous application of osmoprotectants on grain yield $\left(\mathrm{kg} \mathrm{ha}^{-1}\right)$, leaf soluble protein $\left(\mathrm{mg} \mathrm{g}^{-1}\right)$ and leaf $\mathrm{K}^{+}$contents of chickpea.

\begin{tabular}{|c|c|c|c|c|c|c|c|c|}
\hline \multirow{2}{*}{$\begin{array}{l}\text { Chickpea } \\
\text { genotypes }\end{array}$} & \multirow{2}{*}{$\begin{array}{c}\text { Water } \\
\text { stress } \\
\text { level }\end{array}$} & \multirow{2}{*}{$\begin{array}{l}\text { Foliar } \\
\text { spray }\end{array}$} & \multicolumn{2}{|c|}{ Grain yield $\left(\mathrm{kg} \mathrm{ha}^{-1}\right)$} & \multicolumn{2}{|c|}{$\begin{array}{l}\text { Leaf total soluble } \\
\text { protein ( } \mathbf{m g ~ g}^{-1} \text { ) }\end{array}$} & \multicolumn{2}{|c|}{ leaf $\mathrm{K}^{+}$contents } \\
\hline & & & Bahawalpur & Cholistan & Bahawalpur & Cholistan & Bahawalpur & Cholistan \\
\hline \multirow[t]{9}{*}{$\begin{array}{c}\text { Bhakhar } \\
2011\end{array}$} & \multirow[t]{3}{*}{$\begin{array}{c}\text { Well } \\
\text { watered }\end{array}$} & $\begin{array}{l}\text { Distilled } \\
\text { water }\end{array}$ & 1532.7 bd & $1792.1 \mathrm{ac}$ & $1.07 \mathrm{ac}$ & $1.02 \mathrm{a}$ & $49.52 \mathrm{ad}$ & $44.85 \mathrm{ab}$ \\
\hline & & $\begin{array}{l}\text { Glycine } \\
\text { betaine }\end{array}$ & $1928.6 \mathrm{a}$ & $1950.7 \mathrm{a}$ & $1.15 \mathrm{a}$ & $1.11 \mathrm{a}$ & $52.62 \mathrm{ac}$ & 48.28 a \\
\hline & & Proline & $1732.0 \mathrm{ab}$ & 1844.7 ac & $1.09 \mathrm{ac}$ & $1.06 \mathrm{a}$ & $50.30 \mathrm{ad}$ & 46.97 a \\
\hline & \multirow{3}{*}{$\begin{array}{l}\text { Water } \\
\text { stress at } \\
\text { flowering } \\
\text { + pod } \\
\text { formation } \\
\text { + grain } \\
\text { filling } \\
\text { stage }\end{array}$} & $\begin{array}{l}\text { Distilled } \\
\text { water }\end{array}$ & $1165.2 \mathrm{eg}$ & $1261.7 \mathrm{fh}$ & $0.46 \mathrm{~d}$ & $0.43 \mathrm{~b}$ & 47.41 ad & $42.41 \mathrm{ab}$ \\
\hline & & $\begin{array}{l}\text { Glycine } \\
\text { betaine }\end{array}$ & 1537.1 bd & $1527.6 \mathrm{cf}$ & $0.53 \mathrm{~d}$ & $0.50 \mathrm{~b}$ & $51.38 \mathrm{ac}$ & $45.38 \mathrm{ab}$ \\
\hline & & Proline & 1457.5 be & $1427.0 \mathrm{eg}$ & $0.51 \mathrm{~d}$ & $0.46 \mathrm{~b}$ & $50.13 \mathrm{ad}$ & $44.13 \mathrm{ab}$ \\
\hline & \multirow{3}{*}{$\begin{array}{c}\text { Water } \\
\text { stress at } \\
\text { flowering } \\
\text { stage }\end{array}$} & $\begin{array}{l}\text { Distilled } \\
\text { water }\end{array}$ & 1458.5 be & $1468.3 \mathrm{df}$ & $1.08 \mathrm{ac}$ & $1.05 \mathrm{a}$ & $51.65 \mathrm{ac}$ & $41.65 \mathrm{ab}$ \\
\hline & & $\begin{array}{l}\text { Glycine } \\
\text { betaine }\end{array}$ & $1884.8 \mathrm{a}$ & $1862.6 \mathrm{ab}$ & $1.12 \mathrm{ab}$ & $1.08 \mathrm{a}$ & $54.69 a$ & $47.36 \mathrm{a}$ \\
\hline & & Proline & $1689.5 \mathrm{ac}$ & $1760.2 \mathrm{ad}$ & $1.08 \mathrm{ac}$ & $1.06 \mathrm{a}$ & $53.68 \mathrm{ab}$ & $45.01 \mathrm{ab}$ \\
\hline \multirow[t]{10}{*}{ DUSHT } & \multirow[t]{3}{*}{$\begin{array}{c}\text { Well } \\
\text { watered }\end{array}$} & $\begin{array}{l}\text { Distilled } \\
\text { water }\end{array}$ & $1411.3 \mathrm{bf}$ & $1544.4 \mathrm{cf}$ & $0.99 \mathrm{c}$ & $0.97 \mathrm{a}$ & 47.98ad & $39.31 \mathrm{ab}$ \\
\hline & & $\begin{array}{l}\text { Glycine } \\
\text { betaine }\end{array}$ & 1529.9 bd & $1832.6 \mathrm{ac}$ & $1.03 \mathrm{ac}$ & $1.01 \mathrm{a}$ & $52.24 \mathrm{ac}$ & $44.91 \mathrm{ab}$ \\
\hline & & Proline & 1525.1 bd & 1656.3 ae & $1.01 \mathrm{bc}$ & $1.00 \mathrm{a}$ & $51.03 \mathrm{ac}$ & $41.03 \mathrm{ab}$ \\
\hline & \multirow{3}{*}{$\begin{array}{l}\text { Water } \\
\text { stress at } \\
\text { flowering } \\
\text { + pod } \\
\text { formation } \\
\text { + grain } \\
\text { filling } \\
\text { stage }\end{array}$} & $\begin{array}{c}\text { Distilled } \\
\text { water }\end{array}$ & $1029.9 \mathrm{~g}$ & $963.1 \mathrm{~h}$ & $0.43 \mathrm{~d}$ & $0.41 \mathrm{~b}$ & $41.76 \mathrm{~d}$ & $37.16 \mathrm{~b}$ \\
\hline & & $\begin{array}{l}\text { Glycine } \\
\text { betaine }\end{array}$ & $1352.3 \mathrm{dg}$ & $1254.3 \mathrm{fh}$ & $0.49 \mathrm{~d}$ & $0.46 \mathrm{~b}$ & $45.40 \mathrm{bd}$ & $42.07 \mathrm{ab}$ \\
\hline & & Proline & $1123.5 \mathrm{fg}$ & $1129.3 \mathrm{gh}$ & $0.45 \mathrm{~d}$ & $0.43 \mathrm{~b}$ & $44.86 \mathrm{~cd}$ & $41.53 \mathrm{ab}$ \\
\hline & \multirow{3}{*}{$\begin{array}{l}\text { Water } \\
\text { stress at } \\
\text { flowering } \\
\text { stage }\end{array}$} & $\begin{array}{l}\text { Distilled } \\
\text { water }\end{array}$ & $1385.2 \mathrm{cf}$ & $1373.2 \mathrm{eg}$ & $0.99 \mathrm{c}$ & $0.98 \mathrm{a}$ & $48.70 \mathrm{ad}$ & $42.03 \mathrm{ab}$ \\
\hline & & $\begin{array}{l}\text { Glycine } \\
\text { betaine }\end{array}$ & 1628.8 ad & 1580.3 be & $1.02 \mathrm{bc}$ & $1.04 \mathrm{a}$ & $52.43 \mathrm{ac}$ & $45.77 \mathrm{ab}$ \\
\hline & & Proline & 1556.2 bd & $1533.1 \mathrm{cf}$ & $1.01 \mathrm{bc}$ & $1.03 \mathrm{a}$ & $51.43 \mathrm{ac}$ & $43.93 \mathrm{ab}$ \\
\hline & LSD $0.05 p=$ & & 326.67 & 318.00 & 0.1225 & 0.1467 & 8.6519 & 9.2161 \\
\hline
\end{tabular}

LSD $=$ Least significant difference.

stress by maintenance of leaf water status due to enhanced osmotic adjustment and improved photosynthesis, mainly due to a higher stomatal conductance (Iqbal et al., 2008).

Productivity of a crop mainly depends on its effective utilization of available moisture. Application of irrigation water at reproductive stage of chickpea improved the photosynthetic rate and also increased the grain filling period, consequently improving the number of pods per plant, grain weight and ultimately the grain yield (Allu et al., 2014). Conversely, water stress restricted the chickpea productivity by reducing the plant growth, canopy structure as indicated by leaf senescence, photosynthesis, chlorophyll contents that ultimately produced lower photosynthates and inefficient remobilization of the photo-assimilates from source to pods that resulted in short and shriveled grains (Sankar et al., 2007). Exogenous application of GB improved the number of pods per plant, grain weight and grain yield both under water stress and well-watered conditions. Foliar spray of GB improved the productivity by improving the water potential, osmotic potential, 
Table 3. Influence of exogenous application of osmoprotectants on antioxidant enzymes status of chickpea.

\begin{tabular}{|c|c|c|c|c|c|c|c|c|}
\hline \multirow{2}{*}{$\begin{array}{l}\text { Chickpea } \\
\text { genotypes }\end{array}$} & \multirow{2}{*}{$\begin{array}{c}\text { Water } \\
\text { stress } \\
\text { level }\end{array}$} & \multirow{2}{*}{$\begin{array}{l}\text { Foliar } \\
\text { spray }\end{array}$} & \multicolumn{2}{|c|}{$\begin{array}{l}\text { Superoxide dismutase (IU } \\
\text { min }^{-1} \mathbf{m g}^{-1} \text { protein) }\end{array}$} & \multicolumn{2}{|c|}{$\begin{array}{l}\text { Peroxidase }\left(\mathrm{mmol} \mathrm{min}^{-1}\right. \\
\left.\mathrm{mg}^{-1} \text { protein }\right)\end{array}$} & \multicolumn{2}{|c|}{$\begin{array}{l}\text { Catalase }\left(\mu \text { mol } \text { min }^{-1}\right. \\
\text { mg-1 protein })^{-1}\end{array}$} \\
\hline & & & Bahawalpur & Cholistan & Bahawalpur & Cholistan & Bahawalpur & Cholistan \\
\hline \multirow[t]{9}{*}{$\begin{array}{l}\text { Bhakhar } \\
2011\end{array}$} & \multirow[t]{3}{*}{$\begin{array}{c}\text { Well } \\
\text { watered }\end{array}$} & $\begin{array}{l}\text { Distilled } \\
\text { water }\end{array}$ & $72.01 \mathrm{~g}$ & $63.98 \mathrm{ef}$ & 7.29 de & $6.63 \mathrm{de}$ & $40.32 \mathrm{eg}$ & $34.49 \mathrm{fh}$ \\
\hline & & $\begin{array}{l}\text { Glycine } \\
\text { betaine }\end{array}$ & $95.45 \mathrm{e}$ & 74.10 e & $9.57 \mathrm{c}$ & 7.90 de & $48.24 \mathrm{de}$ & $45.47 \mathrm{e}$ \\
\hline & & Proline & 91.19 ef & 69.93 ef & $8.54 \mathrm{~cd}$ & $7.47 \mathrm{de}$ & 45.25 de & $37.98 \mathrm{eh}$ \\
\hline & \multirow{3}{*}{$\begin{array}{l}\text { Water } \\
\text { stress at } \\
\text { flowering } \\
\text { + pod } \\
\text { formation } \\
\text { + grain } \\
\text { filling } \\
\text { stage }\end{array}$} & $\begin{array}{l}\text { Distilled } \\
\text { water }\end{array}$ & 195.95 c & $184.10 \mathrm{c}$ & $11.80 \mathrm{~b}$ & $13.46 \mathrm{~b}$ & 84.31 bc & $77.21 \mathrm{~cd}$ \\
\hline & & $\begin{array}{l}\text { Glycine } \\
\text { betaine }\end{array}$ & $227.23 \mathrm{a}$ & $217.09 \mathrm{a}$ & $13.47 \mathrm{a}$ & $17.23 \mathrm{a}$ & $105.31 \mathrm{a}$ & $101.04 \mathrm{a}$ \\
\hline & & Proline & $214.95 a b$ & $203.62 \mathrm{ab}$ & $12.48 \mathrm{ab}$ & $16.11 \mathrm{a}$ & $102.41 \mathrm{a}$ & $95.97 \mathrm{a}$ \\
\hline & \multirow{3}{*}{$\begin{array}{c}\text { Water } \\
\text { stress at } \\
\text { flowering } \\
\text { stage }\end{array}$} & $\begin{array}{l}\text { Distilled } \\
\text { water }\end{array}$ & $71.58 \mathrm{~g}$ & 67.89 ef & 7.14 de & $6.91 \mathrm{de}$ & $43.80 \mathrm{df}$ & $36.20 \mathrm{eh}$ \\
\hline & & $\begin{array}{l}\text { Glycine } \\
\text { betaine }\end{array}$ & 91.49 e & 73.03 ef & $8.61 \mathrm{~cd}$ & $8.11 \mathrm{de}$ & $52.94 \mathrm{~d}$ & 44.49 ef \\
\hline & & Proline & $81.01 \mathrm{eg}$ & 71.39 ef & $8.47 \mathrm{~cd}$ & $7.88 \mathrm{de}$ & $47.55 \mathrm{de}$ & 42.77 ef \\
\hline \multirow[t]{10}{*}{ DUSHT } & \multirow[t]{3}{*}{$\begin{array}{c}\text { Well } \\
\text { watered }\end{array}$} & $\begin{array}{l}\text { Distilled } \\
\text { water }\end{array}$ & $65.82 \mathrm{~g}$ & $60.15 \mathrm{f}$ & $5.66 \mathrm{e}$ & $6.13 \mathrm{e}$ & $32.29 \mathrm{~g}$ & $28.27 \mathrm{~h}$ \\
\hline & & $\begin{array}{l}\text { Glycine } \\
\text { betaine }\end{array}$ & $78.58 \mathrm{eg}$ & $68.57 \mathrm{ef}$ & $7.26 \mathrm{de}$ & $6.93 \mathrm{de}$ & $42.37 \mathrm{dg}$ & $40.76 \mathrm{eg}$ \\
\hline & & Proline & $70.79 \mathrm{~g}$ & 66.29 ef & $6.32 \mathrm{e}$ & 6.49 de & $40.81 \mathrm{eg}$ & $34.62 \mathrm{fh}$ \\
\hline & \multirow{3}{*}{$\begin{array}{l}\text { Water } \\
\text { stress at } \\
\text { flowering } \\
\text { + pod } \\
\text { formation } \\
\text { + grain } \\
\text { filling } \\
\text { stage }\end{array}$} & $\begin{array}{l}\text { Distilled } \\
\text { water }\end{array}$ & $166.64 \mathrm{~d}$ & $159.88 \mathrm{~d}$ & $9.47 \mathrm{c}$ & $10.15 \mathrm{c}$ & $74.51 \mathrm{c}$ & $71.90 \mathrm{~d}$ \\
\hline & & $\begin{array}{l}\text { Glycine } \\
\text { betaine }\end{array}$ & 207.49 bc & 196.59 bc & $11.63 \mathrm{~b}$ & $13.16 \mathrm{~b}$ & $90.56 \mathrm{~b}$ & $92.05 \mathrm{ab}$ \\
\hline & & Proline & 194.99 c & 184.25 c & $11.29 \mathrm{~b}$ & $12.62 \mathrm{~b}$ & $78.36 \mathrm{c}$ & 82.69 bc \\
\hline & \multirow{3}{*}{$\begin{array}{c}\text { Water } \\
\text { stress at } \\
\text { flowering } \\
\text { stage }\end{array}$} & $\begin{array}{l}\text { Distilled } \\
\text { water }\end{array}$ & $66.86 \mathrm{~g}$ & 62.23 ef & $6.54 \mathrm{e}$ & $6.41 \mathrm{de}$ & $33.03 \mathrm{fg}$ & $30.73 \mathrm{gh}$ \\
\hline & & $\begin{array}{l}\text { Glycine } \\
\text { betaine }\end{array}$ & $74.21 \mathrm{fg}$ & 67.28 ef & $8.42 \mathrm{~cd}$ & 8.10 de & $40.97 \mathrm{eg}$ & 43.44 ef \\
\hline & & Proline & $72.26 \mathrm{~g}$ & 64.73 ef & $8.25 \mathrm{~cd}$ & $8.15 \mathrm{~d}$ & $37.98 \mathrm{eg}$ & $39.34 \mathrm{eg}$ \\
\hline & LSD $0.05 p=$ & & 17.074 & 13.647 & 1.6400 & 1.9988 & 11.118 & 10.374 \\
\hline
\end{tabular}

LSD $=$ Least significant difference.

turgor pressure, water use efficiency that induced water stress tolerance in crop, thus keeping high photosynthetic activities, which then lead to improved proficiency of the plant to distribute more photoassimilates to developing grains and produced higher yield (Hussain et al., 2009).

Water stress significantly reduced the leaf soluble protein and $\mathrm{K}^{+}$contents of chickpea genotypes. However, exogenously applied proline and GB could considerably compensate this loss. Exogenous application of GB produced significantly higher total leaf soluble protein and leaf $\mathrm{K}^{+}$contents. As its application was found to be more effective in ameliorating the negative impact of water stress (Azam et al., 2005). Foliar spray of GB improved the total leaf soluble protein and leaf $\mathrm{K}^{+}$contents of plants subjected to moisture stress by maintenance of leaf water status due to enhanced osmotic adjustment, improved photosynthesis and enhanced root growth that result in higher uptake of nutrients (Iqbal et al., 2008).

Antioxidant protection system plays an imperative role in plant's response to stress situations. The accumulation of ROS was related to be sensed as an 'alarm' signal that initiates pre-emptive defense responses (Shao et al., 2005). In present investigation the antioxidant activities increase in both chickpea cultivars under water stress situation at both locations. Higher antioxidants activities during water stress can protect plants from oxidative injury (Laxa et al., 2019). Under water stress condition, antioxidant enzymes (SOD, POX and CAT) protect the cells from ROS and they 
were found maximum in Bhakhar 2011. Lower activity of antioxidant was found in DUSHT (Zaefyzadeh et al., 2009). Foliar application of GB significantly improved the antioxidant enzyme activities in chickpea plants cultivated under moisture stress. It might be due to the fact that GB application induced enzymatic activities in seedlings particularly SOD and CAT activities and it proved to enhance the cellular integrity and allowed the plants to maintain its water status resulting in improving of photosynthesis and general metabolism activities (Farooq et al., 2008).

\section{Conclusion}

Results of present study showed that water stress flowering + pod formation + grain filling stage of chickpea adversely affects its yield and physiological parameters. However, exogenous application of GB mitigates the adverse effects of water stress and enhanced tolerance in chickpea mainly due to higher antioxidant enzymes activity, demonstrating the protective measures of plant cells in stress condition. Hence, antioxidants status might be a suitable method for illustrating water stress tolerance in chickpea.

\section{References}

ALLU, A.D., SOJA, A.M., WU, A., SZYMANSKI, J. and BALAZADEH, S., 2014. Salt stress and senescence: identification of cross-talk regulatory components. Journal of Experimental Botany, vol. 65, no. 14, pp. 3993-4008. http://dx.doi.org/10.1093/jxb/eru173. PMid:24803504.

ALMESELMANI, M., DESHMUKH, P.S., SAIRAM, R.K., KUSHWAHA, S.R. and SINGH, T.P., 2006. Protective role of antioxidant enzymes under high temperature stress. Plant Science, vol. 171, no. 3 , pp. 382-388. http://dx.doi.org/10.1016/j.plantsci.2006.04.009. PMid:22980208.

ASHRAF, M. and FOOLAD, M.R., 2007. Role of glycine betaine and proline in improving plant abiotic stress resistance. Environmental and Experimental Botany, vol. 59, no. 2, pp. 206-216. http://dx.doi.org/10.1016/j.envexpbot.2005.12.006.

AWARI, V.R., DALVI, U.S., LOKHANDE, P.K., PAWAR, V.Y., MATE, S.N., NAIK, R.M. and MHASE, L.B., 2017. Physiological and biochemical basis for moisture stress tolerance in chickpea under pot study. International Journal of Current Microbiology and Applied Sciences, vol. 6, no. 5, pp. 1247-1259. http://dx.doi. org/10.20546/ijcmas.2017.605.135.

AZAM, F., ASHRAF, M., ASHRAF, M.Y. and IQBAL, N., 2005. Effect of exogenous application of glycine betaine on capitulum size and achene number of sunflower under water stress. International Journal of Biology and Biotechnology, vol. 2, pp. 765-771.

BARDHAN, K., KUMAR, V. and DHIMMAR, S., 2007. An evaluation of the potentiality of exogenous osmoprotectants mitigating water stress on chickpea. Journal of Agricultural Sciences, vol. 3, no. 2, pp. 67-74. http://dx.doi.org/10.4038/jas.v3i2.8101.

BRADFORD, M.M., 1976. A rapid sensitive method for the quantification of microgram quantities of protein utilising the principle of protein-dye binding. Analytical Biochemistry, vol. 72, no. 1-2, pp. 248-254. http://dx.doi.org/10.1016/00032697(76)90527-3. PMid:942051.
CHANCE, B. and MAEHLY, A.C., 1955. Assay of catalase and peroxidase. Methods in Enzymology, vol. 2, pp. 764-775. http:// dx.doi.org/10.1016/S0076-6879(55)02300-8.

CHEN, T.H. and MURATA, N., 2011. Glycine betaine protects plants against abiotic stress: mechanisms and biotechnological applications. Plant, Cell E Environment, vol. 34, no. 1, pp. 1-20. http://dx.doi.org/10.1111/j.1365-3040.2010.02232.x. PMid:20946588.

FAROOQ, M., BASRA, S.M.A., WAHID, A., CHEEMA, Z.A., CHEEMA, M.A. and KHALIQ A., 2008. Physiological role of exogenously applied glycinebetaine to improve drought tolerance in fine grain aromatic rice (Oryza sativa L.). Journal Agronomy \& Crop Science, vol. 194, no. 5, pp. 325-333. http://dx.doi.org/10.1111/j.1439037X.2008.00323.X.

FAROOQ, M., WAHID, A. and LEE, D.J., 2009. Exogenously applied polyamines increase drought tolerance of rice by improving leaf water status, photosynthesis and membrane properties. Acta Physiologiae Plantarum, vol. 31, no. 5, pp. 937-945. http:// dx.doi.org/10.1007/s11738-009-0307-2.

GIANNOPOLITIS, C.N. and RIES, S.K., 1977. Superoxide dismutases II. Purification and quantitative relationship with water-soluble protein in seedlings. Plant Physiology, vol. 59, no. 2, pp. 315318. http://dx.doi.org/10.1104/pp.59.2.315. PMid:16659840.

HAYAT, S., IRFAN, M., WANI, A.S., NASSER, A. and AHMAD, A., 2012. Salicylic acidslocal, systemic or inter systemic regulators? Plant Signaling E Behavior, vol. 7, no. 1, pp. 93-102. http://dx.doi. org/10.4161/psb.7.1.18620. PMid:22301975.

HUSSAIN, M., MALIK, M.A., FAROOQ, M., KHAN, M.B., AKRAM, M. and SALEEM, M.F., 2009. Exogenous glycine betaine and salicylic acid application improves water relations, allometry and quality of hybrid sunflower under water deficit conditions. Journal Agronomy \& Crop Science, vol. 195, no. 2, pp. 98-109. http://dx.doi.org/10.1111/j.1439-037X.2008.00354.X.

IQBAL, N., ASHRAF, M. and ASHRAF, M.Y., 2008. Glycine betaine, an osmolyte of interest to improve water stress tolerance in sunflower (Helianthus annuus L.): water relations and yield. South African Journal of Botany, vol. 74, no. 2, pp. 274-281. http://dx.doi.org/10.1016/j.sajb.2007.11.016.

KAUR, G., KUMAR, S., THAKUR, P., MALIK, J.A., BHANDHARI, K., SHARMA, K. and NAYYAR, H., 2011. Involvement of proline in response of chickpea (Cicer arietinum L.) to chilling stress at reproductive stage. Scientia Horticulturae, vol. 128, no. 3, pp. 174-181. http://dx.doi.org/10.1016/j.scienta.2011.01.037.

KHAITOV, B., KURBONOV, A., ABDIEV, A. and ADILOV, M., 2016. Effect of chickpea in association with Rhizobium to crop productivity and soil fertility. European Journal of Soil Science, vol. 5, pp. 105-112.

LAXA, M., LIEBTHAL, M., TELMAN, W., CHIBANI, K. and DIETZ, K., 2019. The role of the plant antioxidant system in drought tolerance. Antioxidants, vol. 8, no. 4, pp. 94. http://dx.doi. org/10.3390/antiox8040094. PMid:30965652.

MERGA, B. and HAJI, J., 2019. Economic importance of chickpea: production, value, and world trade. Food \& Agriculture, vol. 5, no. 1, pp. 1615718. http://dx.doi.org/10.1080/23311932.20 19.1615718.

MUNNÉ-BOSCH, S. and PENUELAS, J., 2003. Photo-and antioxidative protection, and a role for salicylic acid during drought and recovery in field-grown Phillyrea angustifolia plants. Planta, vol. 217, no. 5, pp. 758-766. http://dx.doi.org/10.1007/s00425003-1037-0. PMid:12698367.

PEIFANG, C., HANGYI, L. and YI, L., 2015. Physiological responses of seedling roots of the desert plant Reaumuria soongorica to drought stress. Acta Pratacultuae Sinica, vol. 24, pp. 72-80. 
RADFORD, P.T., 1967. Growth analysis formulae, their use and abuse. Crop Science, vol. 7, no. 3, pp. 171-175. http://dx.doi. org/10.2135/cropsci1967.0011183X000700030001x.

RAHELEH, R., RAMAZANALI, K., ALI, G., ABDOLREZA, B., FARZANEH, N. and MASOUD, R., 2012. Use of biochemical indices and antioxidant enzymes as a screening technique for drought tolerance in Chickpea genotypes (Cicer arietinum L.). African Journal of Agricultural Research, vol. 7, pp. 5372-5380.

REDDY, A.R. and RAGHAVENDRA, A.S., 2006. Photooxidative stress. In: K.V. MADHAVA RAO, A.S. RAGHAVENDRA and K.J. REDDY, eds. Physiology and molecular biology of stress tolerance in plants. The Nertherlands: Springer. http://dx.doi.org/10.1007/14020-4225-6_6.

RICHARDS, L.A., 1954. Diagnosis and improvement of saline and alkali soils. Soil Science, vol. 78, no. 2, pp. 154. http://dx.doi. org/10.1097/00010694-195408000-00012.

SADAK, M.S. and AHMED, M.R.M., 2016. Physiological role of cyanobacteria and glycine betaine on wheat plant grown under salinity stress. International Journal of Pharm Tech Research, vol. 9, pp. 78-92.

SAIRAM, R.K. and SAXENA, D.C., 2000. Oxidative stress and antioxidants in wheat genotypes: possible mechanism of water stress tolerance. Journal Agronomy \& Crop Science, vol. 184, no. 1, pp. 55-61. http://dx.doi.org/10.1046/j.1439037x.2000.00358.x.

SANKAR, B., JALEEL, C.A., MANIVANNAN, P., KISHOREKUMAR, A., SOMASUNDARAM, R. and PANNEERSELVAM, R., 2007. Effect of paclobutrazol on water stress amelioration through antioxidants and free radical scavenging enzymes in Arachis hypogaea L. Colloids and Surfaces. B, Biointerfaces, vol. 60, no. 2, pp. 229-235. http://dx.doi.org/10.1016/j.colsurfb.2007.06.016. PMid:17764913.

SHAO, H.B., LIANG, Z.S., SHAO, M.A. and SUN, Q., 2005. Dynamic changes of antioxidative enzymes of 10 wheat genotypes at soil water deficits. Colloids and Surfaces. B, Biointerfaces, vol. 42, no. 3-4, pp. 187-195. http://dx.doi.org/10.1016/j. colsurfb.2005.02.007. PMid:15876527.

SINHA, R., IRULAPPAN, V., MOHAN-RAJU, B., SUGANTHI, A. and SENTHIL-KUMAR, M., 2019. Impact of drought stress on simultaneously occurring pathogen infection in field-grown chickpea. Scientific Reports, vol. 9, no. 1, pp. 5577. http://dx.doi. org/10.1038/s41598-019-41463-z. PMid:30944350.

STEEL, R.G.D., TORRIE, J.H. and DEEKEY, D.A., 1997. Principles and procedures of statistics: a biometrical approach. 3rd ed. New York: McGraw Hill Book, pp. 400-428.

WANG, H., ZHANG, L., MA, J., LI, X., LI, Y., ZHANG, R. and WANG, R., 2010. Effects of water stress on reactive oxygen species generation and protection system in rice during grain-filling stage. Agricultural Sciences in China, vol. 9, no. 5, pp. 633-641. http://dx.doi.org/10.1016/S1671-2927(09)60138-3.

WATSON, D.J., 1952. The physiological basis of variation in yield. Annals of Botany, vol. 4, pp. 101-105.

ZAEFYZADEH, M., QULIYEV, R.A., BABAYEVA, S. and ABBASOV, M.A., 2009. The effect of the interaction between genotypes and drought stress on the superoxide dismutase and chlorophyll content in durum wheat landraces. Turkish Journal of Biology, vol. 33, pp. 1-7. 